\title{
Weighing risks and benefits of stress ulcer prophylaxis in critically ill patients
}

\author{
Trupesh Chanpura' and Sachin Yende*2 \\ University of Pittsburgh Department of Critical Care Medicine: Evidence-Based Medicine Journal Club, edited by Sachin Yende
}

\section{Expanded abstract Citation}

Marik PE, Tajender Vasu T, Hirani A, Pachinburavan M: Stress ulcer prophylaxis in the new millennium: a systematic review and meta-analysis. Critical Care Med 2010, 38:11.

\section{Background}

Recent observational studies suggest that bleeding from stress ulceration is extremely uncommon in intensive care unit patients. Furthermore, the risk of bleeding may not be altered by the use of acid suppressive therapy. Early enteral tube feeding (initiated within $48 \mathrm{~h}$ of intensive care unit admission) may account for this observation. Stress ulcer prophylaxis may, however, increase the risk of hospital-acquired pneumonia and Clostridia difficile infection.

\section{Methods}

Objective: A systematic review of the literature to determine the benefit and risks of stress ulcer prophylaxis and the moderating effect of enteral nutrition.

Design:

Data Sources: MEDLINE, Embase, Cochrane Register of Controlled Trials, and citation review of relevant primary and review articles.

Study Selection: Randomized, controlled studies that evaluated the association between stress ulcer prophylaxis and gastrointestinal bleeding. The authors included only those studies that compared a histamine- 2 receptor blocker with a placebo.

Data Extraction: Data were abstracted on study design, study size, study setting, patient population, histamine-2 receptor blocker and dosage used, incidence of clinically

*Correspondence: yendes@upmc.edu

Deprtment of Critical Care Medicine, 606D Scaife Hall, 3550 Terrace Street,

University of Pittsburgh, Pittsburgh, PA 15261, USA

Full list of author information is available at the end of the article significant gastrointestinal bleeding, hospital-acquired pneumonia, mortality, and the use of enteral nutrition.

\section{Results}

Seventeen studies (which enrolled 1836 patients) met the inclusion criteria. Patients received adequate enteral nutrition in three of the studies. Overall, stress ulcer prophylaxis with a histamine-2 receptor blocker reduced the risk of gastrointestinal bleeding (odds ratio 0.47; 95\% confidence interval, $0.29-0.76 ; P<0.002$; Heterogeneity $\left[\mathrm{I}^{2}\right]=44 \%$ ); however, the treatment effect was noted only in the subgroup of patients who did not receive enteral nutrition. In those patients who were fed enterally, stress ulcer prophylaxis did not alter the risk of gastrointestinal bleeding (odds ratio 1.26; 95\% confidence interval, $0.43-$ 3.7). Overall histamine- 2 receptor blockers did not increase the risk of hospital-acquired pneumonia (odds ratio 1.53; 95\% confidence interval, $0.89-2.61 ; P=0.12$; $\left.\mathrm{I}^{2}=41 \%\right)$; however, this complication was increased in the subgroup of patients who were fed enterally (odds ratio $2.81 ; 95 \%$ confidence interval, $1.20-6.56 ; P=0.02$; $I^{2}=0 \%$ ). Overall, stress ulcer prophylaxis had no effect on hospital mortality (odds ratio 1.03; $95 \%$ confidence interval, $0.78-1.37 ; P=0.82$ ). The hospital mortality was, however, higher in those studies $(\mathrm{n}=2)$ in which patients were fed enterally and received a histamine- 2 receptor blocker (odds ratio 1.89; 95\% confidence interval, 1.043.44; $P=0.04, \mathrm{I}^{2}=0 \%$ ). Sensitivity analysis and metaregression demonstrated no relationship between the treatment effect (risk of gastrointestinal bleeding) and the classification used to define gastrointestinal bleeding, the Jadad quality score or the year the study was reported.

\section{Conclusions}

The results of this meta-analysis suggest that, in those patients receiving enteral nutrition, stress ulcer prophylaxis may not be required and, indeed, such therapy may increase the risk of pneumonia and death. However, because no clinical study has prospectively tested the influence of enteral nutrition on the risk of stress ulcer prophylaxis, those findings should be considered exploratory and interpreted with some caution. 


\section{Commentary}

In 1969, Skillman et al. [1] reported a clinical syndrome of lethal "stress ulceration" in seven of 150 (5\%) consecutive intensive care unit (ICU) patients. These patients had in common respiratory failure, hypotension, and sepsis. Subsequent studies confirmed this finding and two meta-analyses published by Cook et al. [2] demonstrated that both histamine-2 receptor blockers (H2RBs) and sucralfate decreased the risk of bleeding from stress ulceration when compared to a placebo. Stress ulcer prophylaxis (SUP) becomes regarded as the standard of care in patients admitted to the intensive Care Unit (ICU), and this intervention is currently endorsed by Surviving sepsis campaign and American Society of Health System Pharmacists (ASHP) guidelines. The universal use of SUP has been reinforced with the adoption of "ventilator bundles." Currently Joint Commission and the Institute for Healthcare Improvement recommend universal SUP as a core "quality" measure for mechanically ventilated patients.

Although the Agency for Healthcare Research and Quality recommends using SUP only in patients on mechanical ventilation and high bleeding risk from coagulopathies, SUP is used in all critically ill patients and even outside the ICU setting. For example, estimates indicate that approximately $90 \%$ of critically ill patients admitted to the ICU receive some form of SUP [3], and up to $52 \%$ of non-ICU patients receive SUP $[4,5]$. SUP is not without risks. Acid suppressive therapy is associated with increased colonization of the upper gastrointestinal tract with potentially pathogenic organisms and may increase the risk of hospital-acquired pneumonia [5]. Furthermore, gastric acid is an important defense against the acquisition of Clostridium difficile spores, and the use of acid suppressive therapy has been linked to an increased risk of Clostridium difficile infection [6-8]. Thus, understanding risks and benefits of SUP is important. For example, patients receiving enteral alimentation have a lower incidence of stress ulceration than unfed patients [9]. Whether routine SUP in patients who receive enteral feeding is beneficial or harmful is not known.

Marik et al. [10] conducted a meta-analysis of 17 randomized clinical trials and postulated that SUP may have no added benefits in ICU patients who receive enteral nutrition. They examined the effect of different SUP regimes on the risk of gastrointestinal bleeding, hospital-acquired pneumonia, and mortality, stratifying the studies based on enteral nutrition.

The meta-analysis included a total of 1836 enrolled between the years 1980 and 2004. Overall, SUP with a H2RB reduced the risk of GI bleeding $(P<0.002)$ but had no effect on mortality. The beneficial effect of SUP was noted only in the subgroup of patients who did not receive enteral nutrition. SUP did not alter the risk of GI bleeding in patients who received enteral nutrition, and these individuals had higher risk of hospital-acquired pneumonia $(P=0.02, \mathrm{n}=9$ studies $)$ and mortality ( $P=0.04, \mathrm{n}=2$ studies).

The results of this meta-analysis suggest that SUP may not be beneficial in patients who are fed enterally. The strength of this review article includes the rigorous attempt to identify all relevant RCTs studies, consider and evaluate for possible confounding factors, such as year of publications, definition of gastrointestinal bleeding, quality of randomized controlled trials, and publication bias. Limitations of this article includes lack of homogeneity in patient population, difference in diagnostic criteria used for major end-points, and only three studies had patients with enteral nutrition.

\section{Recommendation}

SUP is beneficial in high risk patients, including those that are on mechanical ventilation and have coagulopathy. SUP may cause unfavorable outcomes, such as hospitalacquired pneumonia and Clostridium difficile infection, and clinicians must weigh risks and benefits in low-risk patients, such as those who are not requiring mechanical ventilation or are receiving enteral nutrition.

\section{Competing interests}

The authors declare that they have no competing interests.

\section{Author details}

'The Clinical Research, Investigation, and Systems Modeling of Acute IIIness (CRISMA) Center, University of Pittsburgh, Pittsburgh, PA, USA. ²Department of Critical Care Medicine, University of Pittsburgh, Pittsburgh, PA, USA.

Published: 29 October 2012

\section{References}

1. Skillman JJ, Bushnell LS, Goldman H, et al:. Respiratory failure, hypotension, sepsis and jaundice. A clinical syndrome associated with lethal hemorrhage from acute stress ulceration of the stomach. Am J Surg 1969, 117:523-530.

2. Cook DJ, Witt LG, Cook RJ, et al.: Stress ulcer prophylaxis in the critically ill: a metaanalysis. Am J Med 1991; 91:519-527.

3. Daley RJ, Rebuck JA, Welage LS, et al.: Prevention of stress ulceration: Current trends in critical care. Crit Care Med 2004; 32:2008-2013.

4. Janicki T, Stewart S: Stress-ulcer prophylaxis for general medical patients: a review of the evidence. J Hosp Med 2007; 2:86-92.

5. Herzig SJ, Howell MD, Ngo LH, et al:: Acid suppressive medication use and the risk for hospital-acquired pneumonia. JAMA 2009, 301:2120-2128.

6. Cunningham R, Dale B, Undy B, et al.: Proton pump inhibitors as a risk factor for Clostridium difficile diarrhea. J Hosp Infect 2003, 54:243-245.

7. Dial S, Alrasadi K, Manoukian C, et al.: Risk of Clostridium difficile diarrhea among hospital inpatients prescribed proton pump inhibitors: cohort and case-control studies. Can Med Assoc J 2004, 171:33-38.

8. Louie TJ, Meddings J: Clostridium difficile infection in hospitals: risk factors and responses. Can Med Assoc J 2004, 171:45-46.

9. Pingleton SK, Hadzima SK: Enteral alimentation and gastrointestinal bleeding in mechanically ventilated patients. Crit Care Med 1983, 11:13-16.

10. Marik PE, Vasu T, Hirani A, Pachinburavan M: Stress ulcer prophylaxis in the millennium: a systematic review and meta-analysis. Crit Care Med 2010, 38:2222-2228

doi:10.1186/cc11819

Cite this article as: Chanpura T, Yende S: Weighing risks and benefits of stress ulcer prophylaxis in critically ill patients. Critical Care 2012, 16:322. 